\title{
Transition Radiation for Uneven, Limited Surfaces*
}

\author{
S. Reiche, J. B. Rosenzweig, UCLA, Los Angeles, CA 90095, USA
}

\begin{abstract}
In the most common approach to modeling transition radiation, the annihilation of the incident particle with its image particle at the metallic boundary is calculated. This is valid only for ideal planar boundaries, and is not applicable if the surface is modulated, rough or limited in the transverse dimension. In this paper, we show results obtained by replacing the image current with the induced surface charge and surface distributions. Special problems such as a modulated surface are analytically solved and compared to numerical simulation. Implications for experimental use of transition radiation-based beam monitors are discussed.
\end{abstract}

\section{INTRODUCTION}

Because transition radiation (TR) is frequently used for electron beam diagnostic purposes, a fundamental understanding of the process is essential. In the standard model the electric field of the incident electron polarizes a dielectric boundary. The changing polarization emits radiation in the forward and backward direction [1]. The case of a perfectly conducting plane is obtained in the limit of an infinite value for the dielectric constant. Another more heuristic model employs the annihilation of the electron with its mirror particle [2]. Although simple, the model relies on the crude approximation of an instantaneous halt of both particles' motion.

A more sophisticated and physically transparent model is based on a virtual photon picture of the electron's electric field. Due to the introduction of boundary conditions, these photons becomes real, resulting in the observed TR $[3,4]$. This model is a practical improvement on those previously mentioned, as does not rely on the assumption of an infinite conducting plane, although in previous work it has still required assumption of an even surface to match the boundary condition.

To circumvent the limitations of these previous analyses, we present here a model of radiation generation based on induced surface currents [6]. We obtain the current density for the case of an infinite flat surface, and then apply it to the standard formulae with the modified boundary conditions appropriate for describing finite size, or uneven conducting surfaces.

\section{THE TRANSITION RADIATION MODEL}

For simplicity and brevity, we analyze only the case in which an electron strikes the conducting surface at normal

\footnotetext{
* Work supported by DOE grant DE-FG03-92ER40693
}

incidence. The method presented can easily be generalized to deal with cases of arbitrary incidence. To calculate the radiated energy per frequency and solid angle we derive the induced current density on the surface of a perfectly conducting plane for a relativistic electron whose velocity vector is parallel to the normal vector of the plane (the $z$ direction). We employ the method of image particles to obtain the induced surface charge density, by calculating the normal component of the (retarded) electric field at the boundary. The current density

$$
\vec{J}=\frac{q}{2 \pi} \gamma v \frac{\vec{r}}{\left(r^{2}+\gamma^{2} v^{2} t^{2}\right)^{3 / 2}} \delta(z)
$$

is thus obtained from the continuity equation. Here $\gamma$ is the Lorenz factor and $v$ is the electron velocity. Integrating over the parameterized time $t^{\prime}=(\gamma v / r) t$, the energy per frequency and solid angle [5] becomes

$$
W=\frac{\omega^{2} q^{2}}{4 \pi^{4} c^{3}}\left|\int d x d y \frac{\omega}{\gamma v} K_{1}\left(\frac{\omega r}{\gamma v}\right) \vec{n} \times \vec{n} \times \frac{\vec{r}}{r} e^{-i \vec{k} \vec{r}}\right|^{2}
$$

where $\vec{n}$ defines the direction of observation, $\vec{k}=k \hat{n}$ and $k$ is the wavenumber. We align the coordinate system so that $\vec{n}$ lies in the $x z$-plane. The vectors $\vec{n}$ and $\vec{e}_{z}$ enclose the observation angle $\theta$. The vector product $\vec{n} \times \vec{n} \times \vec{r}$ is then expressed as $x \cos \theta \vec{e}_{1}+y \vec{e}_{2}$. The vector $\vec{e}_{1}$ lies in the $x z$-plane and is perpendicular to $\vec{n}$ while $\vec{e}_{2}$ is identical to $\vec{e}_{y}$. The integrated term proportional to $y \vec{e}_{2}$ is either zero or small compared to the first term and is therefore generally neglected. The remaining integral is

$$
W=\frac{\omega^{2} q^{2}}{4 \pi^{4} c^{3}}\left|\int d x d y \frac{\omega}{\gamma v} \frac{x}{r} \cos \theta K_{1}\left(\frac{\omega r}{\gamma v}\right) e^{-i \vec{k} \vec{r}}\right|^{2}
$$

Further calculations are based on this equation. Note that any arbitrary surface given by $z(x, y)$ is expressed by the phase factor in Eq. 3 as $\vec{k} \cdot \vec{r}=k(x \sin \theta+z(x, y) \cos \theta)$. In the following sections we investigate different cases of interest for the conducting surface surface.

\section{THE INFINITE PLANE}

In the case of a infinite plane we can compare the results of the present model to those obtained by previous methods. The $z$-dependence on $x$ and $y$ vanishes and Eq. 3 is easily integrated by using the identity $K_{0}^{\prime}(a)=-K_{1}(a)$ and the cosine transformation of the Bessel function. The result is

$$
W=\frac{q^{2}}{4 \pi^{2} c}\left(\frac{\beta^{2} \sin (2 \theta)}{1-\beta^{2} \cos ^{2}(\theta)}\right)^{2}
$$


and differs to other formulae [2] by substitution of the factor $\sin (2 \theta) / 2$ for $\sin (\theta)$. The $2 \theta$ dependence of the sine function suppresses any radiation at an angle of 90 degrees. This is physically more reasonable because our model excludes any current density component in $z$.

For a relativistic electron $(\beta \approx 1)$ the radiation is sharply peaked around the angle of $\theta=1 / \gamma$ of maximum emission.

\section{THE FINITE PLANE}

To investigate the experimentally relevant effects of a finite conducting plane, we restrict the integration over $x$ and $y$ in Eq. 3 to a finite size. For sake of simplicity we assume a circular area of radius $R$. The complex term $\exp [-i k r \sin \theta \cos \phi]$ in Eq. 3 can then be expanded into a series of Bessel function. The integration over the azimuthal angle $\phi$ yields a non-zero value only for the term proportional to $J_{1}$. The integral thus becomes

$$
W=\frac{\omega^{2} q^{2}}{\pi^{2} c^{3}}\left|\frac{\omega}{\gamma v} \cos \theta \int_{0}^{R} r K_{1}\left(\frac{\omega r}{\gamma v}\right) J_{1}(k r \sin \theta) d r\right|^{2}
$$

With $W_{0}$ as the energy per frequency and solid angle for the infinite plane (Eq. 4) the final result is

$$
\begin{aligned}
W= & W_{0}\left[1-\frac{\omega R}{\gamma v} K_{1}\left(\frac{\omega R}{\gamma v}\right) J_{0}(k R \sin \theta)\right. \\
& \left.-\frac{c \omega R}{\gamma^{2} v^{2} \sin \theta} K_{0}\left(\frac{\omega R}{\gamma v}\right) J_{1}(k R \sin \theta)\right]^{2}
\end{aligned}
$$

In the limit $R \rightarrow \infty$ the Bessel functions cancel each other and the angular distribution is identical to Eq. 4. Note that Eq. 6 omits a small correction factor arising from a virtual photon analysis [4], which is due to violation of the continuity equation at the edge of the conducting boundary.

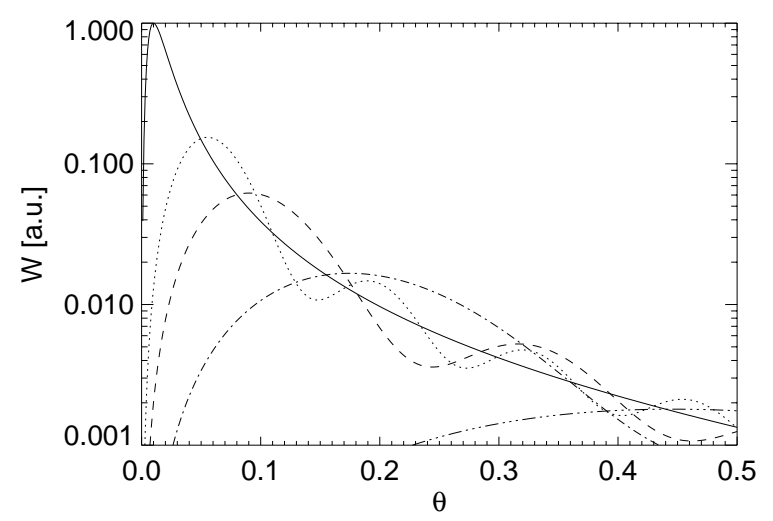

Figure 1: Angular distribution of transition radiation for different radii: $\infty, 10 \mathrm{~mm}, 6.4 \mathrm{~mm}, 3.6 \mathrm{~mm}$ and $1.6 \mathrm{~mm}$ (solid, dotted, dashed, dot-dashed and dot-dot-dot-dashed line, respectively) and $\gamma=100, k=2 \pi / 1 \mathrm{~mm}$.
As seen in Fig. 1 the two main effects of a finite plane boundary are reduced emission, and a wider opening angle due to diffraction. This implies consequences for detectors which rely on a large signal-noise ratio. Although the radiation exhibits diffraction effects due to a small source size of the emitter - namely the finite plane - the opening angle depends on the electron energy as well for a given wavelength.

The argument of the Bessel function $K_{1}$ in Eq. 3 mainly determines the active area of emitting radiation. The exponential drop of $K_{1}$ for large arguments suppress any contribution to the radiation outside $\omega r / \gamma v>6$. Thus we estimate the boundary of the active area as $R_{a} \approx \gamma \lambda$. For coherent transition radiation in the millimeter range it requires targets of the size of several centimeters to achieve maximum signal. The cut-off of coherent transition radiation (CTR) production at long wavelengths has been observed in experiments, and causes complication of interpreting bunch length measurements base on CTR [7].

\section{THE MODULATED PLANE}

A sinusoidally modulated plane can be seen as a first conceptual step towards understanding both grating transition radiation (GTR)[8], and the production of TR from a rough surface. A modulated plane effects only a certain wavelength range. In the limit of long wavelength the phase modulation due to the complex term $\exp [i k z \cos \theta]$ becomes negligible while in the opposite limit the radius of the actively radiation area (s.a.) becomes smaller than one period of the modulation and thus approach the case of a flat plane. The modulation is described by

$$
z=2 \mu \cos [\alpha(x \cos \phi+y \sin \phi)] \cos [\beta(y \cos \phi)-x \sin \phi]
$$

where $\mu$ is the amplitude of the modulation and $\alpha, \beta$ are the wavenumbers in two perpendicular direction within the $x y$-plane. Because the observation point lies within the $x z$-plane we introduce the azimuthal dependence on $\phi$ by rotating the plane instead of the observation point. Eq. 7 is expanded into $\mu\left(\cos \eta_{1}+\cos \eta_{2}\right)$ with $\eta_{1,2}=$ $x[\alpha \cos \phi \mp \beta \sin \phi]+y[\alpha \sin \phi \pm \beta \cos \phi]$. The upper and lower signs denote $\eta_{1}$ and $\eta_{2}$, respectively. Using the identity $\exp [-i a \cos b]=\sum(-i)^{|m|} J_{|m|}(a) e^{i m b}$ the integration of Eq. 3 yields

$$
W=\frac{\omega^{2} q^{2} \cos ^{2} \theta}{\pi^{2} c^{3}}\left|\sum_{m, n} \frac{a_{m n} J_{m n}}{\left(\frac{\omega}{\gamma v}\right)^{2}+a_{m n}^{2}+b_{m n}^{2}}\right|^{2}
$$

with

$$
\begin{aligned}
& J_{m n}=(-i)^{|m|+|n|} J_{|m|}(k \mu \cos \theta) J_{|n|}(k \mu \cos \theta) \\
& a_{m n}=-k \sin \theta+(m+n) \alpha \cos \phi-(m-n) \beta \sin \phi \\
& b_{m n}=(m+n) \alpha \sin \phi+(m-n) \beta \cos \phi
\end{aligned}
$$


In the limit $\omega, \mu \rightarrow 0$ all Bessel functions except for $J_{0}$ become zero. With $J_{00}=1$ the result agrees with Eq. 4. For short wavelength $k \sin \theta$ is the dominant term in $a_{m n}$. By dropping all smaller terms in $a_{m n}$ and $b_{m n}$ only $J_{m n}$ depends on $m$ and $n$. Summing up $J_{m n}$ yields $\exp (-2 i k \mu \cos \theta)$, a phase shift due to the amplitude of the modulation at the impact point of the electron.

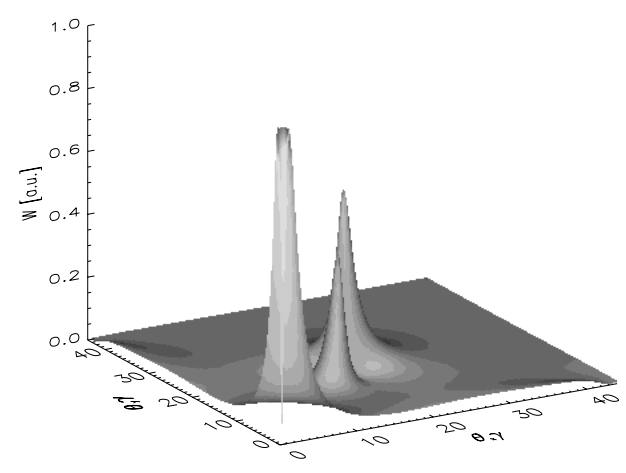

Figure 2: Angular distribution of transition radiation for a modulated plane with $\gamma=35, \lambda=460 \mathrm{~nm}, \mu=100 \mathrm{~nm}$ and $\alpha, \beta=2 \pi / 100 \lambda$.

Fig. 2 shows an example of a diffraction pattern of a modulated surface. Beside the standard emission distribution around $\theta=1 / \gamma$ additional spikes in a well defined direction are present. Those amplitudes diminish for longer wavelengths as their emission angles become larger. Towards shorter wavelength the number of spikes increases and they are grouped closer to the $1 / \gamma$ emission cone.

Notable effects of modulation covers roughly two orders of magnitude around the 'central wavelength' $\mu$. The resonant effects become stronger if the energy of the incident electron is increased, because the effective area of radiation grows and covers more modulation. The resonant overlap becomes stronger. Similar arguments are valid when the period of the modulation is shortened. In these limits, and for modulation in only one transverse direction, the radiation pattern takes on the characteristics of a plane wave reflected from a grating [8].

\section{SURFACE ROUGHNESS}

To study the impact of surface roughness on transition radiation we solve Eq. 3 numerically. We use a recursive algorithm to model roughness where self-similarity is obtained on any zoom level. Based on the results of the previous sections roughness has a larger impact for electrons with higher energy because the active area covers more roughness.

Fig. 3 shows the angular distribution for $\gamma=350, \lambda=$ $100 \mathrm{~nm}$ and a roughness of $100 \mathrm{~nm}$. The transverse size of the bumps are roughly 100 times larger than their height.

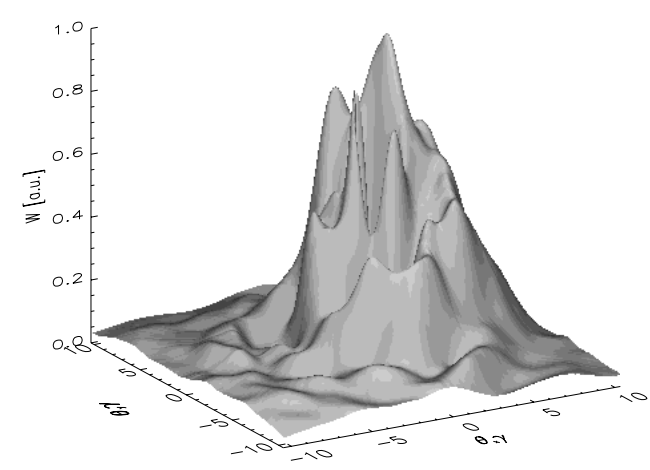

Figure 3: Angular distribution of transition radiation on a rough surface for $\gamma=350$.

In general roughness increases the opening angle of transition radiation for wavelengths shorter than a threshold wavelength. This effect has been observed during the development of optical TR measurements [9] for the VISA FEL experiment. The value of the threshold wavelength depends on the roughness and the electron energy. For high energies, and roughness features small compared to the radiation generation region, the radiation profile has speckle characteristics similar to those obtained from plane wave reflection from a non-uniform mirror.

\section{CONCLUSION}

The model of induced current on a surface generated by an incident electron allows us to calculate the emitted transition radiation distribution and spectrum for a wider range of modeled surfaces. It is important to understand the limits of transition radiation as a tool for beam diagnostics. In particular finite planes and/or rough surfaces reduce the total power and increase the average emission angle reducing the efficiency of applied diagnostics.

\section{REFERENCES}

[1] V.L. Ginzburg and V.N. Tsytovich, Transition Radiation and Transition Scattering (Adam Hilger, Bristol, 1990)

[2] V.L. Ginzburg and I.M. Frank, Journ. Phys. USSR 9 (1945) 353

[3] V.A. Lebedev, Nucl. Instr. \& Meth. A372 (1996) 344

[4] M. Castellano et al.,Nucl. Inst. \& Meth. A435 (1999) 297

[5] J.D. Jackson, Classical Electrodynamics (Wiley, New York, 1975)

[6] J. H. Brownell, J. E. Walsh, and G. Doucas, Phys. Rev. E 57, (1998) 1075.

[7] A. Murokh, et al., Nuclear Instruments and Methods A 410, 549 (1998).

[8] P. Henri, O. Haeberle, P. Rullhusen, N. Maene, W. Mondelaers, Phys. Rev. E 60, (1999) 5.

[9] A. Murokh, et al., these proceedings. 